\title{
Hard X-ray transient grating spectroscopy on bismuth germanate
}

\author{
Jérémy R. Rouxel ${ }^{1,2,3 凶}$, Danny Fainozzi ${ }^{4}$, Roman Mankowsky ${ }^{2}$, Benedikt Rösner ${ }^{2}$, \\ Gediminas Seniutinas ${ }^{2}$, Riccardo Mincigrucci ${ }^{4}$, Sara Catalini ${ }^{5}$, Laura Foglia ${ }^{4}{ }^{4}$, Riccardo Cucini ${ }^{6}$, \\ Florian Döring ${ }^{2}$, Adam Kubec ${ }^{2}$, Frieder Koch², Filippo Bencivenga ${ }^{4}$, Andre Al Haddad², \\ Alessandro Gessini ${ }^{4}$, Alexei A. Maznev ${ }^{7}$, Claudio Cirelli ${ }^{(2}$, Simon Gerber ${ }^{(1)}{ }^{2}$, Bill Pedrini ${ }^{2}$, \\ Giulia F. Mancini ${ }^{12,8}$, Elia Razzoli², Max Burian², Hiroki Ueda ${ }^{2}{ }^{2}$, Georgios Pamfilidis², Eugenio Ferrari², \\ Yunpei Deng2, Aldo Mozzanica², Philip J. M. Johnson (12 2, Dmitry Ozerov², Maria Grazia Izzo ${ }^{9,10}$, \\ Cettina Bottari ${ }^{4}$, Christopher Arrell ${ }^{2}$, Edwin James Divall2 ${ }^{2}$ Serhane Zerdane ${ }^{2}$, Mathias Sander ${ }^{2}$, \\ Gregor Knopp $\mathbb{D}^{2}$, Paul Beaud $\mathbb{D}^{2}$, Henrik Till Lemke ${ }^{2}{ }^{2}$, Chris J. Milne ${ }^{2,11}$, Christian David ${ }^{2}$, \\ Renato Torre ${ }^{5}{ }^{5}$, Majed Chergui ${ }^{1}{ }^{1}$, Keith A. Nelson ${ }^{7}{ }^{7}$, Claudio Masciovecchio ${ }^{4}$, Urs Staub ${ }^{2}$, \\ Luc Patthey ${ }^{2}$ and Cristian Svetina $\mathbb{B}^{2 凶}$
}

\begin{abstract}
Optical-domain transient grating (TG) spectroscopy is a versatile background-free four-wave-mixing technique that is used to probe vibrational, magnetic and electronic degrees of freedom in the time domain 1 . The newly developed coherent $X$-ray free-electron laser sources allow its extension to the $X$-ray regime. $X$-rays offer multiple advantages for TG: their large penetration depth allows probing the bulk properties of materials, their element specificity can address core excited states, and their short wavelengths create excitation gratings with unprecedented momentum transfer and spatial resolution. Here, we demonstrate TG excitation in the hard $X$-ray range at $7.1 \mathrm{keV}$. In bismuth germanate (BGO), the non-resonant TG excitation generates coherent optical phonons detected as a function of time by diffraction of an optical probe pulse. This experiment demonstrates the ability to probe bulk properties of materials and paves the way for ultrafast coherent four-wave-mixing techniques using X-ray probes and involving nanoscale TG spatial periods.

ㅁ1 Four-wave mixing (FWM), in which three coherent pulsed beams with controlled wavelengths, polarizations, wavevectors and arrival times are crossed at the sample ${ }^{2,3}$, is one of the most used optical configurations in time-resolved nonlinear spectroscopies. The nonlinear response of the sample to excitation induced by the crossed light fields generates a fourth beam that emerges with a wavevector determined by the incident wavevectors. A specific outcome of nonlinear spectroscopies is two-dimensional spectros-

copies $^{4}$, which can directly measure matter coherences and disentangle homogeneous and inhomogeneous contributions to spectral lineshapes."
\end{abstract}

A widely used optical configuration in time-resolved nonlinear spectroscopy is the transient grating (TG) geometry (Fig. 1a), in which two simultaneous pulses are crossed in the sample to generate an optical interference pattern that leads to a grating of spatially modulated excitation. A controlled time-delayed probe pulse is then diffracted by this grating. The time dependence of the signal reveals the sample dynamics. The spatial period of the interference pattern is $\Lambda=\lambda / 2 \sin \theta$, where $\lambda$ is the pump wavelength and $2 \theta$ the relative angle between the two pulses. In reciprocal space, the TG wavevector $\mathbf{q}$, with magnitude $q=2 \pi / \Lambda$, is the difference between the two excitation wavevectors $\mathbf{k}_{1}$ and $\mathbf{k}_{2}$. The diffracted signal wavevector $\mathbf{k}_{\mathrm{s}}$ is given by the phase-matching condition $\mathbf{k}_{\mathrm{s}}=\mathbf{k}_{1}-\mathbf{k}_{2}+\mathbf{k}_{3}$, where $\mathbf{k}_{3}$ is the probe beam wavevector. TGs have been used to investigate dispersive excitations such as acoustic waves ${ }^{5}$, phonon-polaritons ${ }^{6}$, transport phenomena in solids, including heat, charge, chemical species, excitons and spins ${ }^{7-10}$. These measurements are often conducted in a range of $\Lambda$ matching the frequency of acoustic waves in the sample ${ }^{11}$. TG can be further used to distinguish between diffusive or ballistic transport as it allows for discrimination between long and short mean free path scattering events in the quasiparticle transport. For example, thermal transport can be readily measured in various materials by a TG following the temporal evolution of the signal. A TG density modulation in a bulk material produces a refractive index change that diffracts the probe light. The diffracted signal intensity diminishes in time as heat moves from the TG peaks to minima, ultimately suppressing the TG pattern and hence the scattered signal. Note that, in the case of an opaque material, for the excitation frequency a TG modulation corresponds to a surface modulation rather than a bulk modulation.

\footnotetext{
'Ecole Polytechnique Fédérale de Lausanne, Laboratory of Ultrafast Spectroscopy (LSU) and Lausanne Centre for Ultrafast Science (LACUS), Lausanne, Switzerland. ${ }^{2}$ Paul Scherrer Institut, Villigen, Switzerland. ${ }^{3}$ Univ. Lyon, UJM-Saint-Etienne, CNRS, Graduate School Optics Institute, Laboratoire Hubert Curien, Saint-Etienne, France. ${ }^{4}$ Elettra-Sincrotrone Trieste, Trieste, Italy. ${ }^{5}$ European Laboratory for Non-Linear Spectroscopy (LENS) and Dip. Di Fisica ed Astronomia, Università degli studi di Firenze, Sesto Fiorentino, Florence, Italy. ${ }^{6}$ Istituto Officina dei Materiali-CNR, Basovizza, Italy. ${ }^{7}$ Department of Chemistry, Massachusetts Institute of Technology, Cambridge, MA, USA. ${ }^{8}$ Department of Physics, University of Pavia, Pavia, Italy. ${ }^{9}$ Sapienza Università di Roma, Dipartimento di Ingegneria informatica, automatica e gestionale Antonio Ruberti, Rome, Italy. ${ }^{10}$ Istituto Italiano di Tecnologia - Center for Life Nanoscience, Rome, Italy. ${ }^{11}$ European XFEL GmbH, Schenefeld, Germany.凶e-mail: jeremy.rouxel@univ-st-etienne.fr; cristian.svetina@psi.ch
} 
a
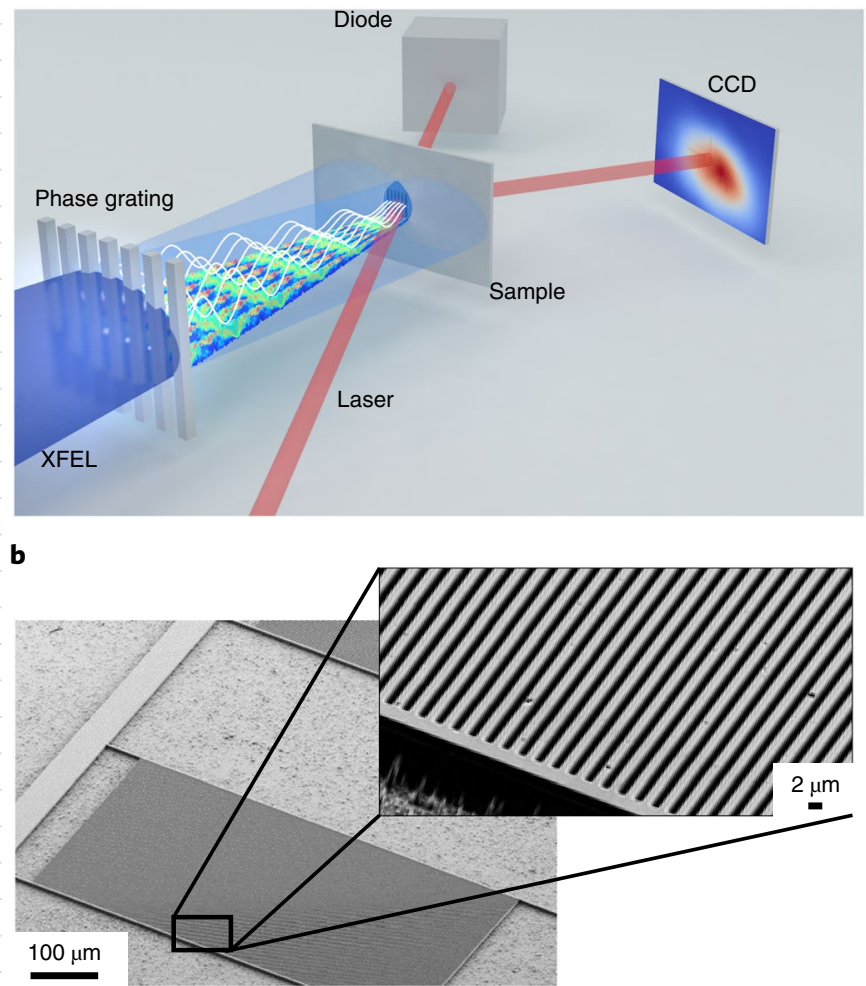

c

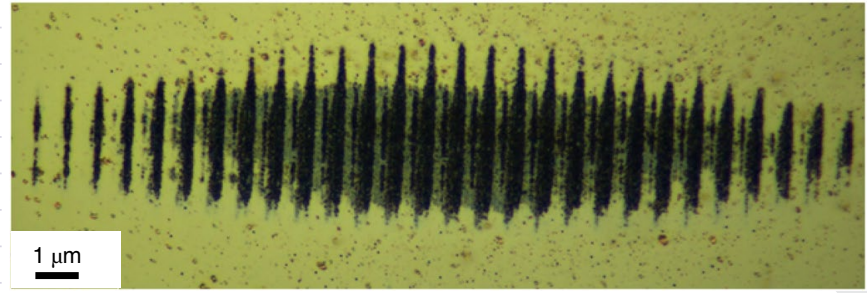

Fig. 1 | Insert text here. a, Schematic of the X-ray TG (XTG) set-up. The incoming XFEL pulse (in blue) is diffracted by a transmission phase grating. Interferences between the diffracted orders generate a Talbot

Q7 carpet, that is, a region of self-images of the originating phase grating. The sample experiences a TG excitation with smaller spatial periodicity due to the convergence of the X-ray beam. A delayed optical pulse (in red) probes the dynamics stimulated by the XTG via transient diffraction, which are detected by an array detector (charge-coupled device, CCD). b, Scanning electron microscopy (SEM) image of a diamond phase grating. $\mathbf{c}$, Permanent grating imprint on a gold surface placed at the sample position and illuminated with an intense XFEL pulse.

In high-quality crystals, thermal transport was observed to be diffusive for TG periods of tens of micrometres, with ballistic contributions leading to deviations from diffusive kinetics at TG periods below $15 \mu \mathrm{m}$ (that is, comparable to the mean free path of some of the heat-carrying acoustic phonons ${ }^{12,13}$ ). At such a spatial range, the TG signal decay timescale $\tau$ due to transport is proportional to the square of the spatial period, which determines the transport length $D$, that is, $D \propto \sqrt{\tau}$. In most materials, submicrometre spatial periods are necessary to observe non-diffusive thermal kinetics, and nanoscale periods are required to resolve the ballistic limit. New information and insights about other transport properties can also be provided by measurements with nanometric TG periods. Gigahertz and terahertz acoustic modes can be observed, even if they are highly damped, as is common at high frequencies in imper- fect or disordered materials. TG periods matching or approaching those of charge or spin-density waves or structural modulations might result in excitation of amplitude or phase modes. There is thus great incentive to develop a nanoscale TG methodology that requires short wavelengths. The availability of X-ray free-electron lasers (XFELs) makes it possible to generate gratings with periods down to a few nanometres. In the hard X-ray region, TG measurements could explore material wavevector ranges currently reached in inelastic X-ray scattering and inelastic neutron scattering ${ }^{14}$. We believe that this will add a complementary tool in many research fields, just as optical TG and FWM measurements have provided a wealth of information that could not be extracted from light scattering methods in the same spectral range.

Recently, TG in the extreme ultraviolet (EUV) regime was successfully demonstrated ${ }^{15,16}$. However, EUV light penetrates matter at most a few tens of nanometres and restricts resonant excitation to light elements. Extending TG into the hard X-ray range (XTG) would allow probing of bulk material behaviour ${ }^{17,18}$, further increasing the range of TG wavevectors that could be reached, as well as giving access to the resonant excitation at the absorption edges of a broader range of elements.

Crossing the beams to generate and probe a TG becomes very challenging in the hard X-ray range. Having recently demonstrated the ability to use the Talbot effect to produce permanent spatial gratings in gold, we demonstrate, here, its use in the weak interaction limit in bismuth germanium oxide, $\mathrm{Bi}_{4} \mathrm{Ge}_{3} \mathrm{O}_{12}$ (BGO). This offers an optimal approach to investigate ultrafast transport with XTG with very high line density, while avoiding the use of a grazing incidence geometry on the reflective optics, which leads to substantial losses and complicated geometries. The experimental layout at the SwissFEL ${ }^{19}$ Bernina endstation ${ }^{20}$ is shown in Fig. 1a. This employs a diamond phase grating with a period of $960 \mathrm{~nm}$ in the X-ray beam path (Fig. 1b). Diamond is used for its resilience to high-intensity X-ray pulses. The diffraction on the grating generates spatially and temporally overlapped phase-locked interfering beams, avoiding the complex procedure of splitting and crossing $\mathrm{X}$-ray beams. A variably delayed optical probe pulse is incident at the Bragg angle for diffraction from the TG material response. The XTG was created using 7.1-keV pulses with duration of $40 \mathrm{fs}$ (r.m.s.) from the SwissFEL ${ }^{19,20}$, without a monochromator. The defocused X-ray beam produced an elliptical $250 \times 150-\mu \mathrm{m}$ (full-width at half-maximum, FWHM) spot at the sample, a room-temperature crystal of BGO. The diamond phase grating was placed $150 \mathrm{~mm}$ upstream of the sample to generate XTG excitation gratings with period of $770 \mathrm{~nm}$, as seen on a gold target, using intensities above the damage threshold, in Fig. 1c. This agrees perfectly with the simulated Talbot carpet pattern. A 400-nm, 80-fs (r.m.s.) laser pulse (3.1 eV, second harmonic of a Ti:sapphire laser) was delivered onto the sample with a spot size of $190 \mu \mathrm{m} \times 150 \mu \mathrm{m}$ (FWHM). The energies of the FEL and probe laser pulses at the sample were $1.5 \mu \mathrm{J}$ and $1.2 \mu \mathrm{J}$, respectively. The diffracted probe beam was recorded by a visible charge-coupled device (CCD) camera mounted on a diffractometer. The signal was obtained by integrating the diffracted light intensity at each probe delay over a detector positioned at the diffraction (phase-matching) angle. Figure 2 shows typical camera images detected before (Fig. 2a), at (Fig. 2b) and $150 \mathrm{fs}$ after (Fig. 2c) the overlap time between the XTG and the probe (time zero). To reduce background, a lens was installed in front of the CCD and the observed spot shape results from both our imaging set-up and the spread due to the detector angle of the sample. Further details on the experimental procedures are provided in the Supplementary Information.

BGO is an optically isotropic material with cubic crystalline structure (eulitine) that is widely used for applications ${ }^{21,22}$ such as scintillation detection for high-energy physics ${ }^{23,24}$, holographic data storage $e^{24}$, high-resolution positron emission tomography ${ }^{25}$, 
a

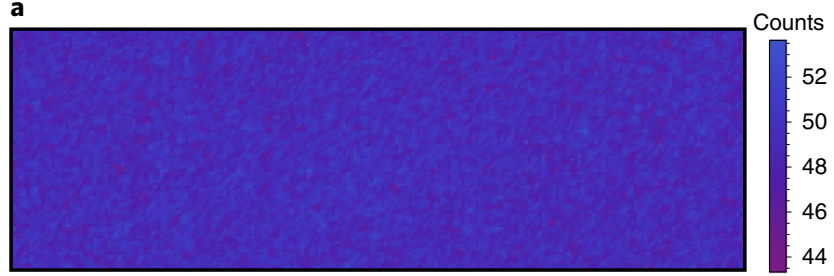

b

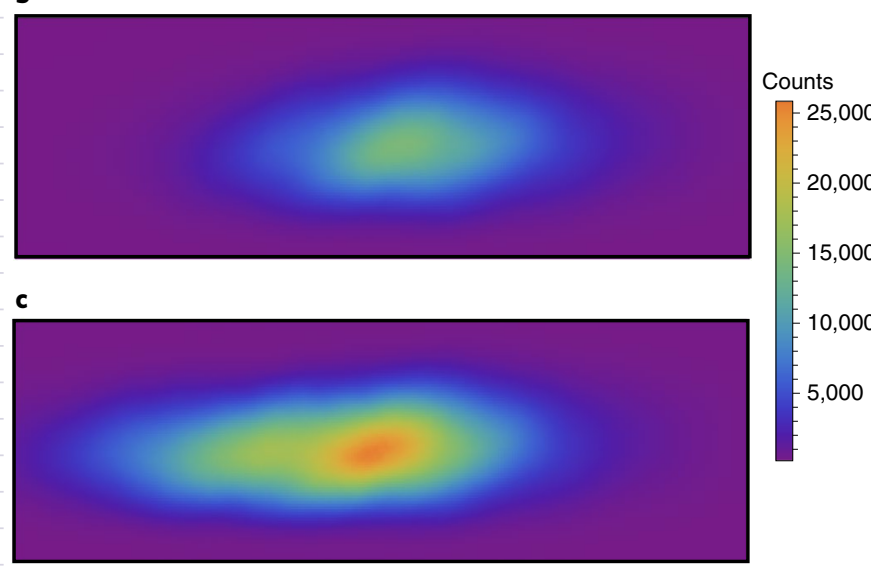

Fig. 2 | Footprints of the signal beam on the CCD detector. $\mathbf{a}$, Before time zero, no signal is observed. b,c, At time zero (b), the XTG signal rises and reaches a maximum after $150 \mathrm{fs}$ (c).

solid-state laser hosting when activated with trivalent rare-earth ions ${ }^{26,27}$ and as an electro-optic material for optical voltage, current and electric power sensors ${ }^{28}$.

Figure 3 shows the dependence of the signal intensity $I_{\mathrm{XTG}}$ on the X-ray pump intensity $I_{\mathrm{FEL}}$ at a probe time delay of $2 \mathrm{ps}$. The quadratic dependence confirms the expected signal nonlinearity ${ }^{5}$ :

$$
I_{\mathrm{XTG}} \propto\left|\chi^{(3)} I_{\mathrm{FEL}} I_{\text {probe }}^{1 / 2} \frac{L}{\lambda} \operatorname{sinc}(\Delta k L / 2)\right|^{2}
$$

where $\chi^{(3)}$ is the effective third-order susceptibility, $I_{\mathrm{FEL}}$ and $I_{\text {probe }}$ are the FEL pump and probe light intensity, respectively, $L$ is the interaction length in the sample, $\lambda$ is the probe wavelength and $\Delta k$ is the phase-matching condition. Our diffraction efficiency is considerably higher than in the EUV regime (for further details see Supplementary Section 5.5) because of the greater penetration depth $(\sim 6 \mu \mathrm{m})$ of hard X-rays (BGO is transparent to the near-UV probe wavelength), highlighting the capability of XTG to measure the bulk properties of matter.

In Fig. $4 a$, the XTG time trace displays a sharp rise at time zero $\left(t_{0}\right)$, followed by oscillations and a decay to a steady-state signal. The non-oscillatory part of the signal can be fit by the following time-dependent form via least-square minimization:

$$
I(t)=\left|\frac{1}{2}\left(1+\operatorname{erf}\left(\frac{t-t_{0}}{\sigma}\right)\right)\left(c_{1} \mathrm{e}^{-\frac{t-t_{0}}{\tau}}+c_{2}\right)\right|^{2}
$$

where $c_{1}$ is the amplitude of the electronic to $A_{1}$ optical phonon mode energy transfer, which decays with $t=970 \pm 30 \mathrm{fs}$, and $c_{2}$ accounts for a thermal signal that is constant on the displayed timescale. The quantity $\sigma=92 \pm 6$ fs is the rise time, reflecting the convolution between the X-ray pulse duration and the probe pulse duration as well as the non-collinear geometry. The periodic oscillation (emphasized in the inset of Fig. $4 \mathrm{~b}$ ) corresponds to the $\mathrm{A}_{1}$ optical phonon mode of BGO at a frequency of $2.6 \pm 0.1 \mathrm{THz}$, which is

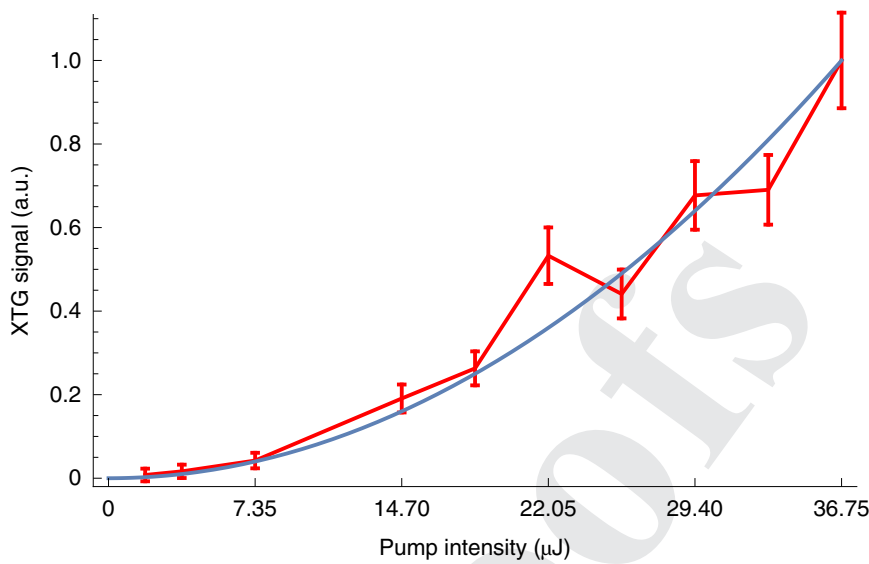

Fig. 3 The XTG signal at 2-ps time delay as a function of the X-ray intensity at the sample. The experimental results (red) are consistent with a quadratic trend (blue).

in very good agreement with the literature ${ }^{29,30}$ and with the phonon dispersion curve at the XTG wavevector (Supplementary Section 3 ). The optical phonon and the single exponential decay of the steady-state signal have also been reported in an EUV experiment ${ }^{31}$. Data extending to a delay of $300 \mathrm{ps}$ are shown in the Supplementary Information and demonstrate $24-\mathrm{GHz}$ oscillations. These oscillations, which are assumed to have a thermal origin, require further investigation. XTG signals are able to follow the material response from tens of femtoseconds to tens of nanoseconds, thus spanning a broad range of physical phenomena.

The relatively simple XTG approach used here can easily be implemented at any XFEL facility on solids or liquids, and to probe a broad range of materials such as magnetic systems, heterostructures, and excitonic systems ${ }^{32}$ such as light-harvesting complexes, to name a few. Indeed, the use of hard X-ray TG offers various advantages compared to optical or EUV TG. First, extremely small excitation grating periods (high TG wavevectors) are now achievable. In the present case, the main limitation is the wavelength of the optical probe, which puts a lower bound on the periodicity of the TG that can be probed. However, a hard X-ray probe can easily detect periods at the nanometric scales or even down to the ångström. Further limitations could arise from the phase grating pattern. Here, nanolithography techniques for the fabrication of diffractive hard X-ray lenses with periods of tens of nanometres have been developed ${ }^{33,34}$. It may also become possible to use superlattice structures for the phase grating ${ }^{35}$ to move to even smaller periods, or to design other means of crossing the X-ray beams such that large angles $\theta$ can be achieved. The present phase grating period range can already provide access to bulk nanoscale heat, charge and spin transport regimes that are difficult to access through conventional methods. The capability for rapidly changing the XTG wavevector by switching the phase grating patterns, as routinely done in optical TG experiments, will enable incisive transport measurements in the nanoscale range where macroscale diffusive kinetics may no longer apply ${ }^{36,37}$. Elemental specificity will be extremely useful for electronic and spin measurements. With the ultrashort pulses of new X-ray sources (few femtoseconds or below), measurements of the coherent relaxation of core-hole states can become accessible, adding valuable information for core excited-state dynamics. The signal sensitivity could also be greatly improved by using self-heterodyne detection schemes that make use of the probe diffracted beam from the phase grating as a local oscillator ${ }^{38,39}$. Finally, the Talbot approach can be readily extended to multi-colour FEL pulses for different excitation and probe wavelengths. This will facilitate the implementation of condensed phase analogues of theoretically pro- 

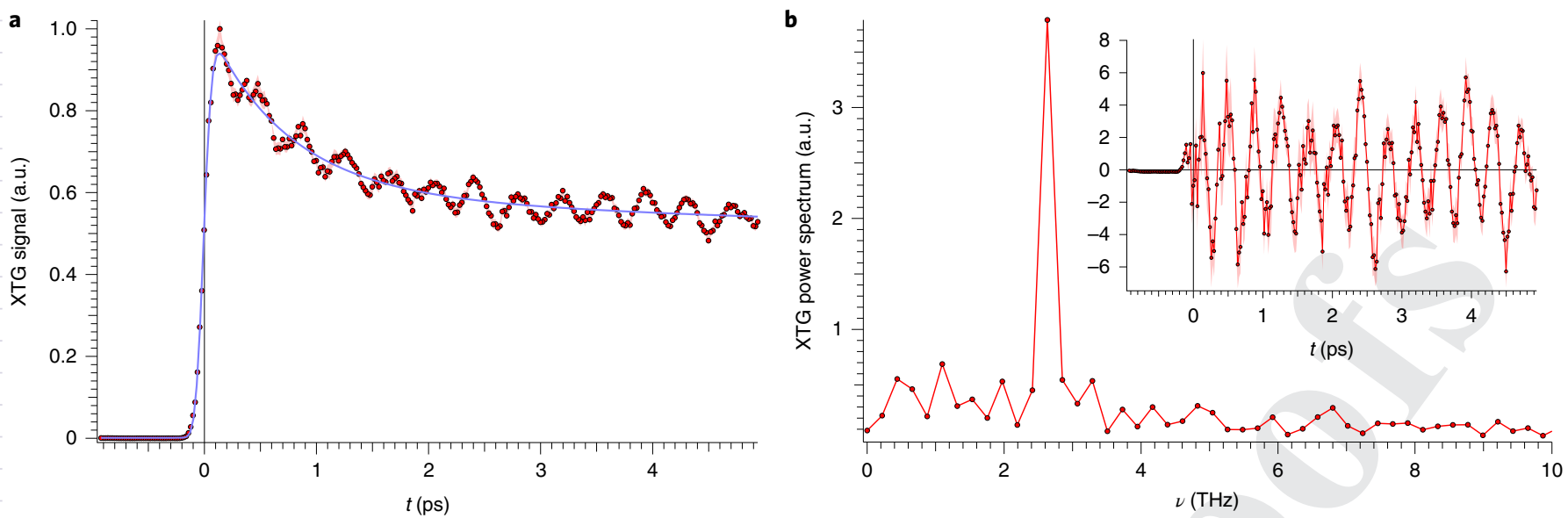

Fig. 4 | XTG signals from BGO at $7.1 \mathrm{keV}$ with an excitation grating pitch of $770 \mathbf{n m}$. $\mathbf{a}$, Time trace at short times ( $<5 \mathrm{ps)}$ normalized to 1 . The

non-oscillatory decay of the signal is fitted (in blue) with equation (2). The fast oscillations are attributed to optical phonon coherence. $\mathbf{b}$, Fourier transform of the fast-oscillatory component (residuals from the fit in a are shown in the inset).

posed methods ${ }^{40}$ for the study of the delocalization of electronic excited states, electronic coherences and charge transfer processes, with ultrafast time resolution and atomic selectivity.

\section{Online content}

Any methods, additional references, Nature Research reporting summaries, source data, extended data, supplementary information, acknowledgements, peer review information; details of author contributions and competing interests; and statements of data and code availability are available at https:/doi.org/10.1038/ s41566-021-00797-9.

Received: 11 December 2020; Accepted: 9 March 2021;

\section{References}

1. Goodno, G. D., Dadusc, G. \& Miller, R. D. Ultrafast heterodyne-detected transient-grating spectroscopy using diffractive optics. J. Opt. Soc. Am. B 15 1791-1794 (1998).

2. Thomson, R., Leburn, C. \& Reid, D. (eds) Ultrafast Nonlinear Optics (Springer, 2013).

3. Mukamel, S. Principles of Nonlinear Optical Spectroscopy 6 (Oxford Univ. Press, 1995).

4. Hamm, P. \& Zanni, M. Concepts and Methods of 2D Infrared Spectroscopy (Cambridge Univ. Press, 2011).

5. Rogers, J. A., Maznev, A. A., Banet, M. J. \& Nelson, K. A. Optical generation and characterization of acoustic waves in thin films: fundamentals and applications. Annu. Rev. Mater. Sci. 30, 117-157 (2000).

6. Crimmins, T. F., Stoyanov, N. S. \& Nelson, K. A. Heterodyned impulsive stimulated Raman scattering of phonon-polaritons in $\mathrm{LiTaO}_{3}$ and $\mathrm{LiNbO}_{3}$. J. Chem. Phys. 117, 2882-2896 (2002).

7. Redman, D. A. et al. Spin dynamics and electronic states of N-V centers in diamond by EPR and four-wave-mixing spectroscopy. Phys. Rev. Lett. 67, 3420-3423 (1991)

8. West, B. A., Womick, J. M. \& Moran, A. M. Probing ultrafast dynamics in adenine with mid-UV four-wave mixing spectroscopies. J. Phys. Chem. A 115, 8630-8637 (2011)

9. Dhar, L., Rogers, J. A. \& Nelson, K. A. Time-resolved vibrational spectroscopy in the impulsive limit. Chem. Rev. 94, 157-193 (1994).

10. Janušonis, J. et al. Transient grating spectroscopy in magnetic thin films: simultaneous detection of elastic and magnetic dynamics. Sci. Rep. 6, 29143 (2016)

11. Tobey, R. I. et al. Transient grating measurement of surface acoustic waves in thin metal films with extreme ultraviolet radiation. Appl. Phys. Lett. 89, 091108 (2006).

12. Johnson, J. A. et al. Direct measurement of room-temperature nondiffusive thermal transport over micron distances in a silicon membrane. Phys. Rev. Lett. 110, 025901 (2013).

13. Hua, C. \& Minnich, AustinJ. Transport regimes in quasiballistic heat conduction. Phys. Rev. B 89, 094302 (2014).
14. Behrens, C. et al. Few-femtosecond time-resolved measurements of X-ray free-electron lasers. Nat. Commun. 5, 3762 (2014).

15. Bencivenga, F. et al. Four-wave mixing experiments with extreme ultraviolet transient gratings. Nature 520, 205-208 (2015).

16. Bencivenga, F. et al. Nanoscale transient gratings excited and probed by extreme ultraviolet femtosecond pulses. Sci. Adv. 5, eaaw5805 (2019).

17. Schweigert, I. V. \& Mukamel, S. Coherent ultrafast core-hole correlation spectroscopy: X-ray analogues of multidimensional NMR. Phys. Rev. Lett. 99, 163001 (2007)

18. Svetina, C. et al. Towards X-ray transient grating spectroscopy. Opt. Lett. 44, 574-577 (2019).

19. Milne, C. J. et al. SwissFEL: the Swiss X-ray free electron laser. Appl. Sci. 7, 720 (2017).

20. Ingold, G. et al. Experimental station Bernina at SwissFEL: condensed matter physics on femtosecond time scales investigated by X-ray diffraction and spectroscopic methods. J. Synchrotron Radiat. 26, 874-886 (2019).

21. Raymond, S. G. \& Townsend, P. D. The influence of rare-earth ions on the low-temperature thermoluminescence of $\mathrm{Bi}_{4} \mathrm{Ge}_{3} \mathrm{O}_{12}$. J. Phys. Condens. Matter 12, 2103-122 (2000).

22. Williams, P. A. et al. Optical, thermo-optic, electro-optic and photoelastic properties of bismuth germanate $\left(\mathrm{Bi}_{4} \mathrm{Ge}_{3} \mathrm{O}_{12}\right)$. Appl. Opt. 35, 3562-3569 (1996).

23. Kaminskii, A. A. et al. Growth, spectral and luminescence study of cubic $\mathrm{Bi}_{4} \mathrm{Ge}_{3} \mathrm{O}_{12}: \mathrm{Pr}^{3+}$ crystals. Phys. Status Solidi A 85, 553-567 (1984).

24. Brunner, S. E. \& Schaart, D. R. BGO as a hybrid scintillator/Cherenkov radiator for cost-effective time-of-flight PET. Phys. Med. Biol. 62, 4421-4439 (2017)

25. Tao, L., Coffee, R. N., Jeong, D. \& Levin, C. S. Ionizing photon interactions modulate the optical properties of crystals with femtosecond scale temporal resolution. Phys. Med. Biol. 66, 045032 (2021).

26. Kamada, O. \& Kakishita, K. Electro-optical effect of $\mathrm{Bi}_{4} \mathrm{Ge}_{3} \mathrm{O}_{12}$ crystals for optical voltage sensors. Jpn J. Appl. Phys. 32, 4288-4291 (1993).

27. Kaminskii, A. A. et al. Growth, spectroscopy and stimulated emission of cubic $\mathrm{Bi}_{4} \mathrm{Ge}_{3} \mathrm{O}_{12}$ crystals doped with $\mathrm{Dy}^{3+}, \mathrm{Ho}^{3+}, \mathrm{Er}^{3+}, \mathrm{Tm}^{3+}$ or $\mathrm{Yb}^{3+}$ ions. Phys. Status Solidi A 56, 725-736 (1979).

28. Li, C. \& Yoshino, T. Simultaneous measurement of current and voltage by use of one bismuth germanate crystal. Appl. Opt. 41, 5391-5397 (2002).

29. Chen, Z., Gao, Y., Minch, B. C. \& DeCamp, M. F. Coherent optical phonon generation in $\mathrm{Bi}_{3} \mathrm{Ge}_{4} \mathrm{O}_{12}$. J. Phys. Condens. Matter 23, 385402 (2011).

30. Couzi, M., Vignalou, J. R. \& Boulon, G. Infrared and Raman study of the optical phonons in $\mathrm{Bi}_{4} \mathrm{Ge}_{3} \mathrm{O}_{12}$ single crystal. Solid State Commun. 20, 461-465 (1976).

31. Maznev, A. A. et al. Generation of coherent phonons by coherent extreme ultraviolet radiation in a transient grating experiment. Appl. Phys. Lett. 113, 221905 (2018)

32. Norman, P. \& Dreuw, A. Simulating X-ray spectroscopies and calculating core-excited states of molecules. Chem. Rev. 118, 7208-7248 (2018).

33. Miao, H., Gomella, A. A., Chedid, N., Chen, L. \& Wen, H. Fabrication of 200-nm period hard X-ray phase gratings. Nano Lett. 14, 3453-3458 (2014).

34. Vila-Comamala, J. et al. Ultra-high resolution zone-doubled diffractive X-ray optics for the multi-keV regime. Opt. Express 19, 175-184 (2011). 
35. Lynch, S. K. et al. Fabrication of $200-n m$ period centimeter area hard X-ray absorption gratings by multilayer deposition. J. Micromech. Microeng. 22, 105007 (2012).

36. Cho, J., Hwang, T. Y. \& Zewail, A. H. Visualization of carrier dynamics in $\mathrm{p}(\mathrm{n})$-type GaAs by scanning ultrafast electron microscopy. Proc. Natl Acad. Sci. USA 111, 2094-2099 (2014).

37. Gorfien, M. et al. Nanoscale thermal transport across an GaAs/AlGaAs heterostructure interface. Struct. Dyn. 7, 025101 (2020).

38. Grilj, J. et al. Self-referencing heterodyne transient grating spectroscopy with short wavelength. Photonics 2, 392-401 (2015).
39. Katayama, K., Yamaguchi, M. \& Sawada, T. Lens-free heterodyne detection for transient grating experiments. Appl. Phys. Lett. 82, 2775-2777 (2003).

40. Tanaka, S. \& Mukamel, S. Coherent X-ray Raman spectroscopy: a nonlinear local probe for electronic excitations. Phys. Rev. Lett. 89, 043001 (2002).

Publisher's note Springer Nature remains neutral with regard to jurisdictional claims in published maps and institutional affiliations.

(c) The Author(s), under exclusive licence to Springer Nature Limited 2021 


\section{Methods}

Experimental set-up. The incident X-ray beam is focused with bendable Kirkpatrick-Baez (KB) mirrors and impinges on a phase grating. The Talbot effect creates a periodic pattern (Talbot carpet) at periodic distances (Talbot distances) through the interference of the diffracted orders. Moreover, the convergence of the $\mathrm{X}$-ray beam ensures that the excitation grating has a smaller pitch than the phase grating (demagnification factor) and this pitch is controlled by the X-ray focusing, the distance between the focus and the phase grating, and the relative distance between the grating and sample. For a $\pi / 2$-phase grating, the transmitted zeroth order is present; it also induces some interaction in the sample, but it does not contribute to the diffracted XTG signal due to the phase-matching condition. This effect is not present in a perfect $\pi$-phase grating.

In our experiment, the gratings and the sample were mounted on the General-Purpose Station of the Bernina endstation at SwissFEL with a separation of $150 \mathrm{~mm}$. For a $\pi$-phase grating of $1,650-\mathrm{nm}$ pitch, this configuration led to an excitation grating with pitch of $\sim 660 \mathrm{~nm}$ at the sample position. In this case, the relative separation of the Talbot planes in the sample area was $\sim 5 \mathrm{~mm}$.

X-ray pump-beam preparation. High-intensity horizontally polarized X-ray pulses were delivered by SwissFEL with a time duration of $\sim 40 \mathrm{fs}$ (r.m.s.) and a repetition rate of $50 \mathrm{~Hz}$. The FEL was tuned to $7.1 \mathrm{keV}$, and the emitted radiation had a bandwidth of $\sim 0.3 \%$ and was used without a monochromator (pink beam condition) for all BGO measurements. The FEL beam was focused on a scintillator coupled with a CCD camera through a microscope (X-ray eye) $750 \mathrm{~mm}$ downstream of the grating, by tuning the curvature of the focusing $\mathrm{KB}$ mirrors. The vertical focus was then adjusted to finally obtain a horizontal strip of excitation $(250 \mathrm{~mm} \times 150 \mathrm{~mm})$ on the sample matching approximately the size of the non-collinear projection of the optical probe on the sample surface at all phase-matching angles. The beam intensity provided by the SwissFEL ranged from $300 \mathrm{~mJ}$ to $800 \mathrm{~mJ}$ and the intensity at the sample was $\sim 1.5 \mathrm{~mJ}$. The phase gratings in the X-ray path were tilted to adjust their effective heights and then match the desired phase shift condition.

Optical probe preparation. The optical probe laser was generated from a Ti:sapphire laser delivering $35-\mathrm{fs}$ pulses at $800 \mathrm{~nm}(10 \mathrm{~mJ})$. A barium borate crystal was used to generate the second harmonic $(400 \mathrm{~nm})$ with an intensity of $\sim 1.2 \mathrm{~mJ}$. A bandpass filter (40-nm bandwidth) was used to remove the unwanted fundamental harmonic and a waveplate was used to control the intensity. Further filtering was passively done by several bandpass reflecting mirrors. The spot size at the sample was tuned to $190 \times 150 \mathrm{~mm}^{2}$ (FWHM) by means of a lens. The arrival time of the probe laser was tuned by a delay stage upstream of the sample and the final reflection to the sample was carried out by a D-shaped mirror to accommodate small phase-matching angles. This D-shaped mirror was mounted on a linear stage (Fig. 2) to change the phase-matching angle when different gratings were being used. The time overlap between the X-ray pump and the optical probe was readjusted for every phase-matching angle.

Diffraction gratings fabrication. Phase gratings were made of polycrystalline Q10 chemical-vapour-deposited diamond (Diamond Materials) and used to excite transient gratings in the samples. The gratings were fabricated using an approach similar to that reported by Makita and colleagues ${ }^{41}$. Ten-millimetre-thick diamond membranes supported by a silicon frame were first cleaned in an $\mathrm{H}_{2} \mathrm{SO}_{4}: \mathrm{H}_{2} \mathrm{O}_{2} 2: 1$ solution at $120^{\circ} \mathrm{C}$ to remove any organic contamination. The membranes were then sputter-coated with a 10 -nm-thick $\mathrm{Cr}$ layer and subsequently spin-coated with a 1-mm-thick negative tone resist (FOX16) followed by baking at $100^{\circ} \mathrm{C}$ for $3 \mathrm{~min}$ on a hotplate. The resist was patterned in an electron-beam lithography system (Raith EBPG 5000PlusES) using an accelerating voltage of $100 \mathrm{kV}$. After the exposure, the samples were developed for $8 \mathrm{~min}$ in Microposit 351:H20 1:3 solution at room temperature, then rinsed in deionized (DI) water and isopropanol. The patterned resist gratings were hard-baked at $300^{\circ} \mathrm{C}$ for $1 \mathrm{~h}$ on a hotplate to increase etch selectivity between the resist and diamond. The unmasked $\mathrm{Cr}$ layer was removed in $\mathrm{Cl}_{2} / \mathrm{O}_{2}$ plasma, revealing the underlying diamond for subsequent

Q11 etching. Finally, the hydrogen silsesquioxane grating pattern was transferred into the diamond by oxygen plasma etching in an Oxford PlasmaLab 100 machine with the following etching parameters: chamber pressure of 10 mtorr, 30-s.c.c.m. $\mathrm{O}_{2}$ flow rate, powers of inductively coupled plasma (ICP) and radiofrequency (RF) of $750 \mathrm{~W}$ and $100 \mathrm{~W}$, respectively. After the etching, the remaining mask was stripped in $10 \% \mathrm{HF}$ solution and the samples were cleaned in $\mathrm{Cr}$ etchant and $\mathrm{H}_{2} \mathrm{SO}_{4}: \mathrm{H}_{2} \mathrm{O}_{2}$ 2:1 solution, followed by rinsing in DI water and isopropanol.

Detectors. The optical beams were measured by a CCD PCO-edge camera. A $2 f-2 f$ lens (half distance of $200 \mathrm{~mm}$ ) was installed in front of the CCD imaging the sample at the chip. This allowed us to reduce the background at the detector on the CCD camera. To control the angle of detection for different phase-matching angles, the CCD camera was mounted on a heavy-load $\delta-\gamma$ diffractometer at $\sim 800 \mathrm{~mm}$ from the sample position and moved in the diffraction plane of the experiment. The $q$ angle was scanned to locate the diffracted XTG signal near the calculated value. The results for a typical diffracted XTG spot recorded with the detector are displayed in Fig. 4. The signal amplitude was obtained by integrating over the signal area, and the weak background was subtracted using an area where the signal was absent. This weak background originated from the isotropic scattering of the optical beam from the sample. Dark shots (unpumped signal) were recorded too and used for normalizing the data as well as to check that the sample was not printed during the measurements. Finally, an ultrafast diode was positioned in transmission along the optical probe path with the purpose to measure the laser transmitted through the sample.

\section{Data availability}

The raw data used in this study are available from the corresponding authors upon request.

\section{References}

41. Makita, M. et al. Fabrication of diamond diffraction gratings for experiments with intense hard X-rays. Microelectron. Eng. 176, 75-78 (2017).

\section{Acknowledgements}

This study was supported by the Swiss National Science Foundation (SNSF, grant no. 200021_165550/1), the SNSF research instrument NCCR Molecular Ultrafast Science and Technology (NCCR MUST, grants 51NF40-183615 and 200021_169017), the ERC Grant 'DYNAMOX' (ERC-2015-AdG-694097) and the EU-H2020 Research and Innovation Programme under the Marie Skłodowska-Curie grant agreements (701647, 654360 NFFA-Europe, 801459-FP-RESOMUS and 871124 Laserlab-Europe). The contribution of the MIT participants A.A.M. and K.A.N. was supported by the US Department of Energy award DE-SC0019126. We thank M. Dzambegovic for the graphical rendering of Fig. la.

\section{Author contributions}

C.S. conceptualized the framework of the experiment. J.R.R. and C.S. designed the experiment. G.S. and C.D. fabricated the diamond gratings. B.R. carried out the optical microscopy of the static printed gratings. All members of the team participated in the experiment and were involved in the discussions. J.R.R., D.F. and E.F. carried out the data reduction. J.R.R., D.F. and C.S. performed the data analysis. C.S., J.R.R. and D.F. wrote the manuscript.

\section{Competing interests}

The authors declare no competing interests.

\section{Additional information}

Supplementary information The online version contains supplementary material available at https://doi.org/10.1038/s41566-021-00797-9.

Correspondence and requests for materials should be addressed to J.R.R. or C.S.

Peer review information Nature Photonics thanks Ryan Coffee and the other, anonymous, reviewer(s) for their contribution to the peer review of this work.

Reprints and permissions information is available at www.nature.com/reprints.

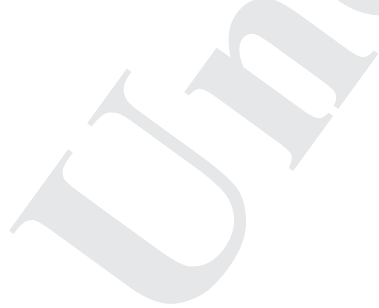




\section{QUERY FORM}

\section{Nature Photonics}

\begin{tabular}{|l|c|}
\hline Manuscript ID & [Art. Id: 797] \\
\hline Author & Jérémy R. Rouxel \\
\hline
\end{tabular}

\section{AUTHOR:}

The following queries have arisen during the editing of your manuscript. Please answer by making the requisite corrections directly in the e.proofing tool rather than marking them up on the PDF. This will ensure that your corrections are incorporated accurately and that your paper is published as quickly as possible.

\begin{tabular}{|c|c|}
\hline Query No. & Nature of Query \\
\hline Q1: & $\begin{array}{l}\text { Please check that the abstract is OK as edited. Please note that journal style does not allow any text that could be } \\
\text { considered a claim of priority (such as the first, the largest/highest/fastest etc.), so we have removed "for the first } \\
\text { time" from the abstract. }\end{array}$ \\
\hline Q2: & $\begin{array}{l}\text { Please check your article carefully, coordinate with any co-authors and enter all final edits clearly in the eproof, } \\
\text { remembering to save frequently. Once corrections are submitted, we cannot routinely make further changes to } \\
\text { the article. }\end{array}$ \\
\hline Q3: & $\begin{array}{l}\text { Note that the eproof should be amended in only one browser window at any one time; otherwise changes will be } \\
\text { overwritten. }\end{array}$ \\
\hline Q4: & $\begin{array}{l}\text { Author surnames have been highlighted. Please check these carefully and adjust if the first name or surname is } \\
\text { marked up incorrectly. Note that changes here will affect indexing of your article in public repositories such as } \\
\text { PubMed. Also, carefully check the spelling and numbering of all author names and affiliations, and the corre- } \\
\text { sponding email address(es). }\end{array}$ \\
\hline Q5: & $\begin{array}{l}\text { You cannot alter accepted Supplementary Information files except for critical changes to scientific content. If } \\
\text { you do resupply any files, please also provide a brief (but complete) list of changes. If these are not considered } \\
\text { scientific changes, any altered Supplementary files will not be used, only the originally accepted version will be } \\
\text { published. }\end{array}$ \\
\hline Q6: & $\begin{array}{l}\text { If applicable, please ensure that any accession codes and datasets whose DOIs or other identifiers are mentioned } \\
\text { in the paper are scheduled for public release as soon as possible, we recommend within a few days of submitting } \\
\text { your proof, and update the database record with publication details from this article once available. }\end{array}$ \\
\hline Q7: & $\begin{array}{l}\text { Figure legends should begin with a brief title for the whole figure as the first sentence, then continue with a cap- } \\
\text { tion containing a short description of each panel and the symbols used. Please provide the missing title/caption } \\
\text { for this figure } 1 \text {. }\end{array}$ \\
\hline Q8: & $\begin{array}{l}\text { Reference [18] was not originally cited in the text. Please check and confirm that the citation of this reference in } \\
\text { the sentence beginning 'Extending TG into the hard X-ray range' is ok, or suggest an alternative location for it to } \\
\text { be cited. }\end{array}$ \\
\hline Q9: & Inset of Figure $4 \mathrm{~b}$ - please provide a label for the vertical axis. \\
\hline Q10: & Sentence beginning "Phase gratings were made of" - please check that CVD has been expanded correctly. \\
\hline
\end{tabular}




\section{QUERY FORM}

\begin{tabular}{|l|c|}
\hline \multicolumn{2}{|c|}{ Nature Photonics } \\
\hline Manuscript ID & [Art. Id: 797] \\
\hline Author & Jérémy R. Rouxel \\
\hline
\end{tabular}

\section{AUTHOR:}

The following queries have arisen during the editing of your manuscript. Please answer by making the requisite corrections directly in the e.proofing tool rather than marking them up on the PDF. This will ensure that your corrections are incorporated accurately and that your paper is published as quickly as possible.

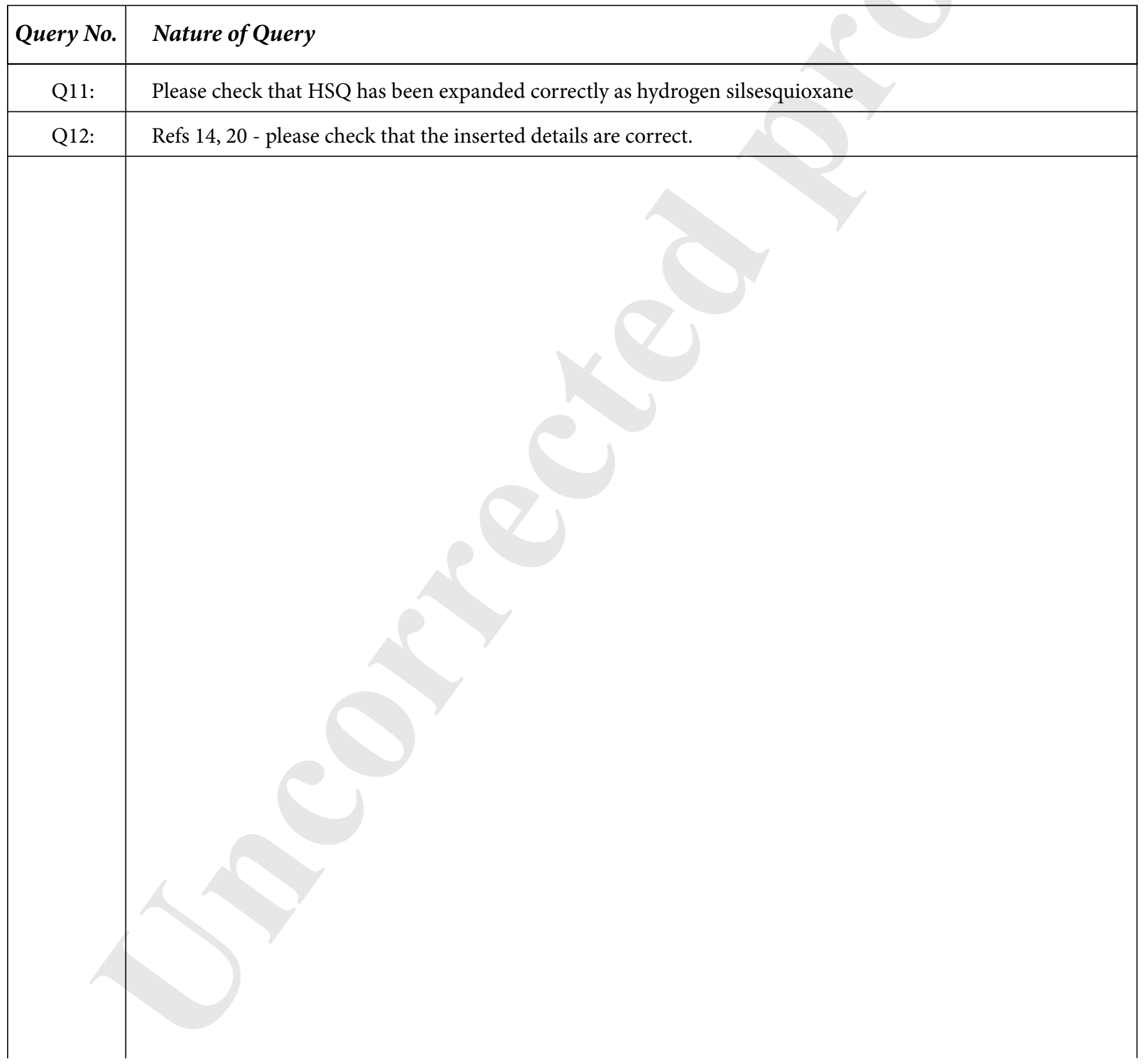

